\title{
Fast Byzantine Gathering with Visibility in Graphs
}

\author{
Avery Miller and Ullash Saha \\ University of Manitoba, Winnipeg, MB, Canada
}

\begin{abstract}
We consider the gathering task by a team of $m$ synchronous mobile robots in a graph of $n$ nodes. Each robot has an identifier (ID) and runs its own deterministic algorithm, i.e., there is no centralized coordinator. We consider a particularly challenging scenario: there are $f$ Byzantine robots in the team that can behave arbitrarily, and even have the ability to change their IDs to any value at any time. There is no way to distinguish these robots from non-faulty robots, other than perhaps observing strange or unexpected behaviour. The goal of the gathering task is to eventually have all non-faulty robots located at the same node in the same round. It is known that no algorithm can solve this task unless there at least $f+1$ non-faulty robots in the team. In this paper, we design an algorithm that runs in polynomial time with respect to $n$ and $m$ that matches this bound, i.e., it works in a team that has exactly $f+1$ non-faulty robots. In our model, we have equipped the robots with sensors that enable each robot to see the subgraph (including robots) within some distance $H$ of its current node. We prove that the gathering task is solvable if this visibility range $H$ is at least the radius of the graph, and not solvable if $H$ is any fixed constant.
\end{abstract}

\section{Introduction}

Mobile robots play a vital role in real-life applications such as military surveillance, search-andrescue, environmental monitoring, transportation, mining, infrastructure protection, and autonomous vehicles. In networks, the robots/agents move from one location to another to collectively complete a task, and might all need to meet at one location in order to share information or start their next task. Therefore, gathering becomes a fundamental problem for mobile robots in networks.

Gathering is hard to accomplish even in a fault-free system, as the robots may not have any planned location where to meet, nor any initial information about the topology of the network. Moreover, in a distributed system, each robot runs its own deterministic algorithm to make decisions, i.e., there is no centralized coordinator. We want a deterministic algorithm that can be run by each robot, and eventually, they will gather at a single node which is not fixed in advance. Additionally, we consider a particularly challenging scenario in which some of the robots are Byzantine: such robots do not follow our installed algorithm and can behave arbitrarily. We can think of these robots as malicious robots in our system, i.e., they have been compromised by outsiders/hackers, and, knowing the algorithm we intend to run, they can behave in ways that attempt to mislead the non-faulty robots into making incorrect decisions. Moreover, non-faulty robots do not know which of the robots (or even how many of the robots) are Byzantine, because all robots look identical. We might face this type of scenario in real-world applications when attackers try to disrupt the normal behavior of systems, so algorithms that are resilient to such attacks are very useful. 
The relative number of non-faulty robots versus Byzantine robots is an essential factor in solving this problem. If there are many Byzantine robots compared to the number of non-faulty robots, then the behaviour of the Byzantine robots can be very influential. As shown in previous work [10], a team that contains $f$ Byzantine robots cannot solve gathering if the number of non-faulty robots is less than $f+1$. The challenge, and the goal of our work, is to provide an efficient gathering algorithm that works when this bound is met, i.e., when the number of non-faulty robots is exactly $f+1$. We provide such an algorithm in a model in which each robot is endowed with sensors that allow them to see all nodes and robots within a fixed distance $H$ of its current location, where $H$ is at least the radius of the network. We also prove an impossibility result which shows that no algorithm can solve gathering in this model if $H$ is any fixed constant (i.e., independent of any graph parameter). It's important to note that this impossibility result does not contradict previous results [4, 5, 10, 19] that provide gathering algorithms with no visibility, as those algorithms make assumptions about additional information known to the robots (such as bounds on the network size, or on the number of Byzantine robots) or make assumptions about additional features such as authenticated whiteboards at the nodes.

\subsection{Model and Definitions}

We consider a team of $m$ robots that are initially placed at arbitrary nodes of an undirected connected graph $G=(V, E)$. We denote by $n$ the number of nodes in the graph, i.e., $n=|V|$. The nodes have no labels. At each node $v$, the incident edges are labeled with port numbers $0, \ldots, \operatorname{deg}(v)-1$ in an arbitrary way, where $\operatorname{deg}(v)$ represents the degree of node $v$. The two endpoints of an edge need not be labeled with the same port number.

For any two nodes $v, w$, the distance between $v$ and $w$, denoted by $d(v, w)$, is defined as the length of a shortest path between $v$ and $w$. The eccentricity of a node $v$, denoted by ecc $(v)$, is the maximum distance from $v$ to any other node, i.e., $\operatorname{ecc}(v)=\max _{w \in V}\{d(v, w)\}$. The radius of a graph, denoted by $R$, is defined as the minimum eccentricity taken over all nodes, i.e., $R=\min _{v \in V}\{\operatorname{ecc}(v)\}$.

The team of $m$ robots contains $f$ Byzantine robots and $m-f$ non-faulty robots. Each robot $\alpha$ has a distinct identifier (ID) $l_{\alpha}$, and it knows its own ID. The Byzantine and non-faulty robots look identical, i.e., there is no way to distinguish them other than perhaps noticing strange or unexpected behaviour. All robots have unbounded memory, i.e., they can remember all information that they have previously gained during their algorithm's execution. We describe the differences between the two types of robots below.

\subsubsection{Properties of non-faulty robots.}

The non-faulty robots have no initial information about the size or topology of the graph, and they have no information about the number of Byzantine robots. A non-negative integer parameter $H$ defines the visibility range of each robot, which we describe in Partial Snapshot below. Each nonfaulty robot executes a synchronous deterministic algorithm: in each round, each robot performs one Look-Compute-Move sequence, i.e., it performs the following three operations in the presented order.

1. The Look operation: A non-faulty robot $\alpha$ located at a node $v$ at the start of round $t$ gains information from two types of view. 
- Local View: Robot $\alpha$ can see the degree of node $v$ and the port numbers of its incident edges. It can also see any other robots located at $v$ at the start of round $t$, along with their ID numbers.

- Partial Snapshot View: Robot $\alpha$ sees the subgraph consisting of all nodes, edges, and port numbers that belong to paths of length at most $H$ that have $v$ as one endpoint. Also, for each node $w$ in this subgraph, robot $\alpha$ sees the list of all IDs of the robots occupying $w$ at the start of round $t$.

2. The Compute operation: Using the information gained during all previous Look operations, a robot $\alpha$ located at a node $v$ deterministically chooses a value from the set $\{n u l l, 0, \ldots, \operatorname{deg}(v)-1\}$. In particular, it chooses null if it decides that it will stay at its current node $v$, and it chooses a value $p \in\{0, \ldots, \operatorname{deg}(v)-1\}$ if it decides to move to the neighbour of node $v$ that is the other endpoint of the incident edge labeled with port number $p$.

3. The Move operation: A robot $\alpha$ located at a node $v$ performs the action that it chose during the Compute operation. In particular, it does nothing if it chose value null, and otherwise, it moves towards a neighbour $w$ of $v$ along the incident edge labeled with the chosen port number $p$, and it arrives at $w$ at the start of the next round. It sees the port number that it uses to enter node $w$. There is no restriction of how robots move along an edge, i.e., multiple robots may traverse an edge simultaneously, in either direction.

All non-faulty robots wake up at the same time and perform their Look-Compute-Move sequences synchronously in every round.

\subsubsection{Properties of the Byzantine robots.}

We assume that a centralized adversary controls all of the Byzantine robots. This adversary has complete knowledge of the algorithm being executed by the non-faulty robots, and can see the entire network and the positions of all robots at all times. In each round, the adversary can make each Byzantine robot move to an arbitrary neighbouring node. Further, we assume that the faulty robots are strongly Byzantine, which means that the adversary can change the ID of any Byzantine robot at any time (in contrast, a weakly Byzantine robot would have a fixed ID during the entire execution).

\subsubsection{Problem Statement.}

Assume that $m$ robots are initially placed at nodes of a network, where $f$ of the robots are strongly Byzantine. The robots synchronously execute a deterministic distributed algorithm. Eventually, all non-faulty robots must terminate their algorithm in the same round, and at termination, all non-faulty robots must be located at the same node.

\subsection{Related Work}

The study of algorithms for mobile robots is extensive, as evidenced by a recent survey [13. The Gathering problem has been investigated thoroughly under a wide variety of model assumptions, as summarized in [3, 9, 12] for continuous models and in [8, 18] for discrete models. Of particular 
interest to our current work are discrete models where the robots are located in a network, have some amount of visibility beyond its own position [1, 2, 17, 11, 15], and where faults may occur [6, 16, 17].

Most relevant to our current work are the results about Gathering in networks when some of the robots can be Byzantine [4, 5, 10, 19]. In [19, the authors consider weakly Byzantine agents and add authenticated whiteboards to the model. Additionally, each robot has the ability to write "signed" messages that authenticate the ID of the writer and whether the message was originally written at the current node. The authors provide an algorithm such that all correct robots gather at a single node in $O(f \cdot|E|)$ rounds, where $f$ is an upper bound on the number of Byzantine robots and $|E|$ is the number of edges in the network.

For the model we consider in our work (but with visibility range 0), the Gathering problem was first considered in [10]. The authors explored the gathering problem under four variants of the model: (i) known size of the graph, weakly Byzantine robots, (ii) known size of the graph, strongly Byzantine robots, (iii) unknown size of the graph, weakly Byzantine robots, and (iv) unknown size of the graph, strongly Byzantine robots. In all cases, the authors assume that the upper bound $f$ on the number of Byzantine robots is known to all non-faulty robots. The authors provided a deterministic polynomial-time algorithms for the two models with weakly Byzantine robots. In the model when the size of the graph is known, their algorithm works for any number of non-faulty robots in the team. Recently, the authors of [14] provided a significantly faster algorithm under the assumption that the number of non-faulty robots in the team is at least $4 f^{2}+8 f+4$. In [10], assuming that the size of the graph is unknown and $f$ robots are weakly Byzantine, the authors provide an algorithm that works when the number of non-faulty robots in the team is $f+2$. They prove a matching lower bound in this scenario: no algorithm can solve Gathering if the number of non-faulty robots in the team is less than $f+2$. For the model with strongly Byzantine robots and known graph size, the authors provided a randomized algorithm that guarantees that the agents gather in a finite number of rounds, and with high probability terminates in $n^{c f}$ rounds for some constant $c>0$. They also provided a deterministic algorithm whose running time is exponential in $n$ and the largest ID belonging to a non-faulty agent. In both cases, the number of non-faulty robots in the team is assumed to be at least $2 f+1$. The authors also proved a lower bound for this model: no algorithm can solve Gathering if the number of non-faulty robots in the team is less than $f+1$. Finally, for the model with strongly Byzantine robots and unknown graph size, they provided a deterministic algorithm that works when the number of non-faulty robots in the team is at least $4 f+2$. The running time is exponential in $n$ and the largest ID belonging to a non-faulty agent. They also proved a lower bound in this model: no algorithm can solve Gathering if the number of non-faulty robots in the team is less than $f+2$. Subsequent work focused on the case of strongly Byzantine robots and attempted to close the gaps between the known upper and lower bounds on the number of non-faulty robots in the team. This was achieved in [4, as the authors provided algorithms that work when the number of non-faulty robots in the team are $f+1$ and $f+2$ for the cases of known and unknown graph size, respectively. However, the running times of these algorithms were also exponential in $n$ and the largest ID belonging to a non-faulty agent.

More recently, the authors of [5] considered a version of the above model that does not assume knowledge of the graph size nor the upper bound $f$ on the number of strongly Byzantine agents. Instead, they considered the amount of initial knowledge as a resource to be quantitatively measured as part of an algorithm's analysis. In this model, they designed an algorithm whose running time is polynomial in $n$ and the number of bits in the smallest ID belonging to a non-faulty agent, 
where $O(\log \log \log n)$ bits of initial information is provided to all robots. The initial information they provide is the value of $\log \log n$, which the algorithm uses as a rough estimate of the graph size. Their algorithm works as long as the number of non-faulty robots in the team is at least $5 f^{2}+6 f+2$. They also proved a lower bound on the amount of initial knowledge: for any deterministic polynomial Gathering algorithm that works when the number of non-faulty robots in the team is at least $5 f^{2}+6 f+2$ and whose running time is polynomial in $n$ and the number of bits in the smallest ID, the amount of initial information provided to all robots must be at least $\Omega(\log \log \log n)$ bits.

\subsection{Our Results}

We consider a graph-based model in which each robot has no initial information other than its own ID and has some visibility range $H$. We prove that no algorithm can solve Gathering in the presence of Byzantine robots if $H$ is any fixed constant. We also design an algorithm that solves Gathering in any graph with $n$ nodes containing $m$ robots, $f$ of which are strongly Byzantine, and where each non-faulty robot has visibility range $H$ equal to the radius of the graph (or larger). Our algorithm has the following desirable properties: (1) the number of rounds is polynomial with respect to $n$ and $m$, in contrast to several previous algorithms whose running times are exponential in $n$ and the largest robot ID; (2) it works when the number of non-faulty robots in the team is $f+1$ (or larger), which is optimal due to an impossibility result from [10] that also holds in our model, and significantly improves on the best previous polynomial-time algorithm, which requires at least $5 f^{2}+6 f+2$ non-faulty robots; (3) it does not assume any initial global knowledge, in contrast to previous algorithms that assume a known bound on the graph size or on the number of Byzantine robots. Such assumptions might be unrealistic in many applications.

\section{The Algorithm}

First, we define some notation that will be used in the algorithm's description and analysis. For any graph $G$, the center of graph $G$ is the set of all nodes that have minimum eccentricity, i.e., all nodes $v \in V(G)$ such that $\operatorname{ecc}(v)=R$, and the center graph of a graph $G$, denoted by $C(G)$, is defined as the subgraph induced by the center nodes. The following terminology will be used to refer to what a robot $\alpha$ can observe in the Look operation of any round $t$ during the execution of an algorithm. The local view at a node $v$ for round $t$ is denoted by Lview $(v, t)$, and refers to all of

the following information: the degree of $v$, the port numbers of its incident edges, and a list of the IDs of all other robots located at node $v$ at the start of round $t$. The snapshot view at a node $v$ for round $t$ is denoted by Sview $(v, t)$, and refers to all of the following information: the subgraph consisting of all nodes, edges, and port numbers that belong to paths of length at most $H$ that have $v$ as one endpoint, and, for each node $w$ in this subgraph, the list of IDs of all robots occupying $w$ at the start of round $t$. For any graph $G$, an ID $l$ is called a singleton ID if the total number of times that $l$ appears as a robot ID at the nodes of $G$ is exactly 1.

\subsection{Algorithm Description}

In what follows, we assume that the visibility range of a non-faulty robot is at least equal to the radius of the graph, i.e., $H \geq R$. We also assume that the number of non-faulty robots is at least $f+1$. 
The algorithm's progress can be divided into three parts. The first part makes each non-faulty robot move to a node $v_{\max }$ such that the robot's snapshot view from $v_{\max }$ contains all the nodes of the network $G$. This is the purpose of our Find-Lookout subroutine, which we now describe. Each robot $\alpha$ produces a list of potential nodes in its initial snapshot view where it thinks it might be located, and it does this by comparing its local view with the degree and robot list of each node in its initial snapshot. It cannot be sure of its initial position within its snapshot view since Byzantine robots can forge $\alpha$ 's ID and position themselves at other nodes that have the same degree as $\alpha$ 's current node. From each guessed initial position, $\alpha$ computes a port sequence of a depth-first traversal of its snapshot view and tries following it in the real network. Since one of the guessed initial positions must be correct, at least one of the depth-first traversals will successfully visit all nodes contained in $\alpha$ 's initial snapshot view. Since the visibility range is at least the radius of the network, the robot's initial snapshot view must contain a node in the center of the network $G$, so at least one step of at least one of the traversals will visit a node in the center of $G$. When located at such a node, the robot will see all nodes in the network. So, by counting how many nodes it sees at every traversal step, and keeping track of where it saw the maximum, it can correctly remember and eventually go back to a node $v_{\max }$ from which it saw all nodes in the network. See Algorithm 11 for the pseudocode of Find-Lookout. After returning to $v_{\max }$ at the end of Find-Lookout, each robot $\alpha$ constructs a set $P_{\alpha}$ consisting of nodes in its snapshot view that match its local view. These can be thought of as 'candidate' locations where $\alpha$ thinks it might actually be located within its snapshot view.

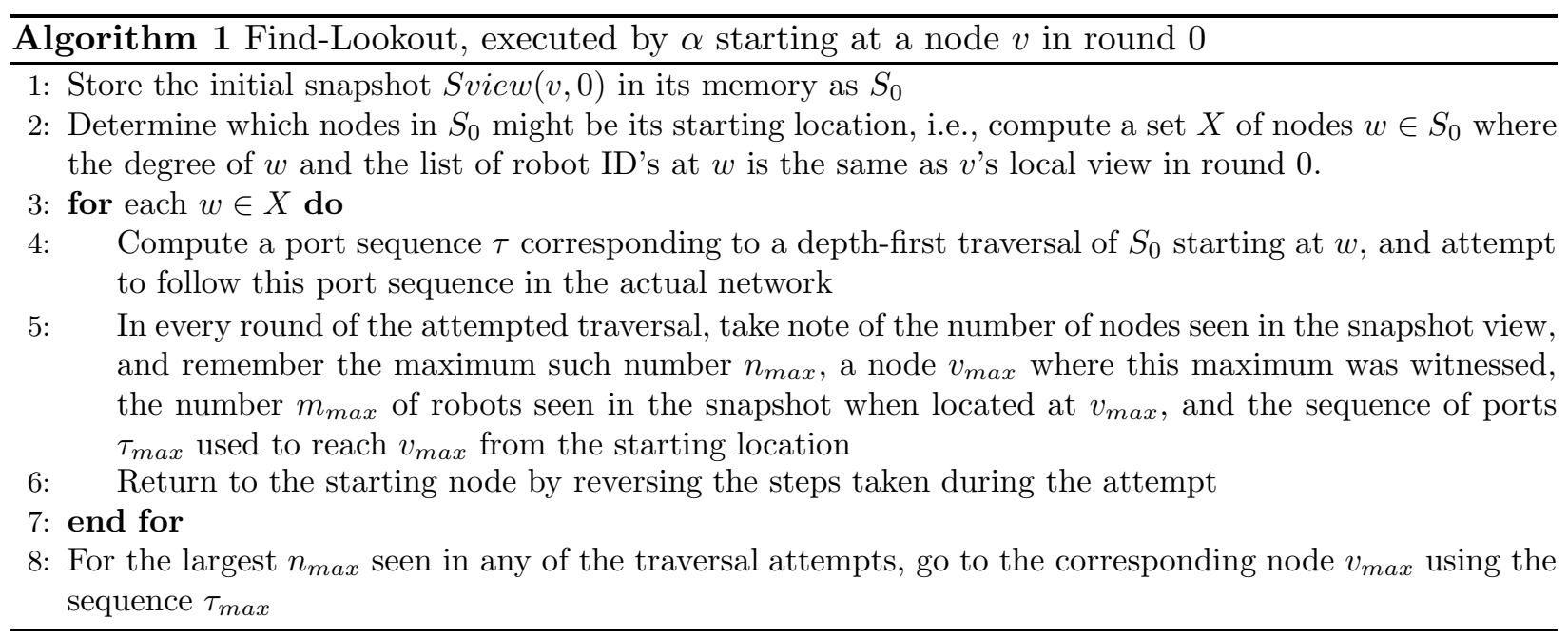

The second part of the algorithm ensures that, eventually, there is a robot with a singleton ID that is located in the center of the network $G$. This is the purpose of our March-to-Center subroutine, which depends highly on the fact that each robot starts this part of the algorithm at a node $v_{\max }$ from which it can see every node in the network. If a robot starts March-to-Center knowing where in its snapshot view it is located (i.e., $\left|P_{\alpha}\right|=1$ ), then the robot moves directly to the center of $G$ : it computes the center of its snapshot, and moves to one of the nodes in the center of this snapshot, which is also the center of the entire network $G$. If all robots do this, then the center of the network will contain a singleton ID, since there are more non-faulty robots than Byzantine robots, and all non-faulty robots have distinct ID's. The difficult case is when a robot $\alpha$ is not sure where in its snapshot it is located at the start of March-to-Center (i.e., $\left|P_{\alpha}\right|>1$ ). This is because 
the Byzantine robots can forge $\alpha$ 's ID and position themselves at other nodes with the same degree as $\alpha$ 's current node. In this case, $\alpha$ will not move during March-to-Center, and simply watch to see if it can spot any inconsistencies between its local view and its possible starting locations in its snapshot. The key observation, which we will prove, is that at least one of the following must happen in each execution of March-to-Center: there is a robot with a singleton ID located at a node in the center of the network, or, at least one robot sees an inconsistency and narrows down its list of possible starting locations. So, after enough repetitions of March-to-Center, we can guarantee that there will be a robot with a singleton ID that is located in the center of the network. The location of the robot with the smallest such singleton ID is chosen as $v_{\text {target }}$ by all non-faulty robots, and this is the place where the robots will eventually gather. See Algorithm 2 for the pseudocode of March-to-Center.

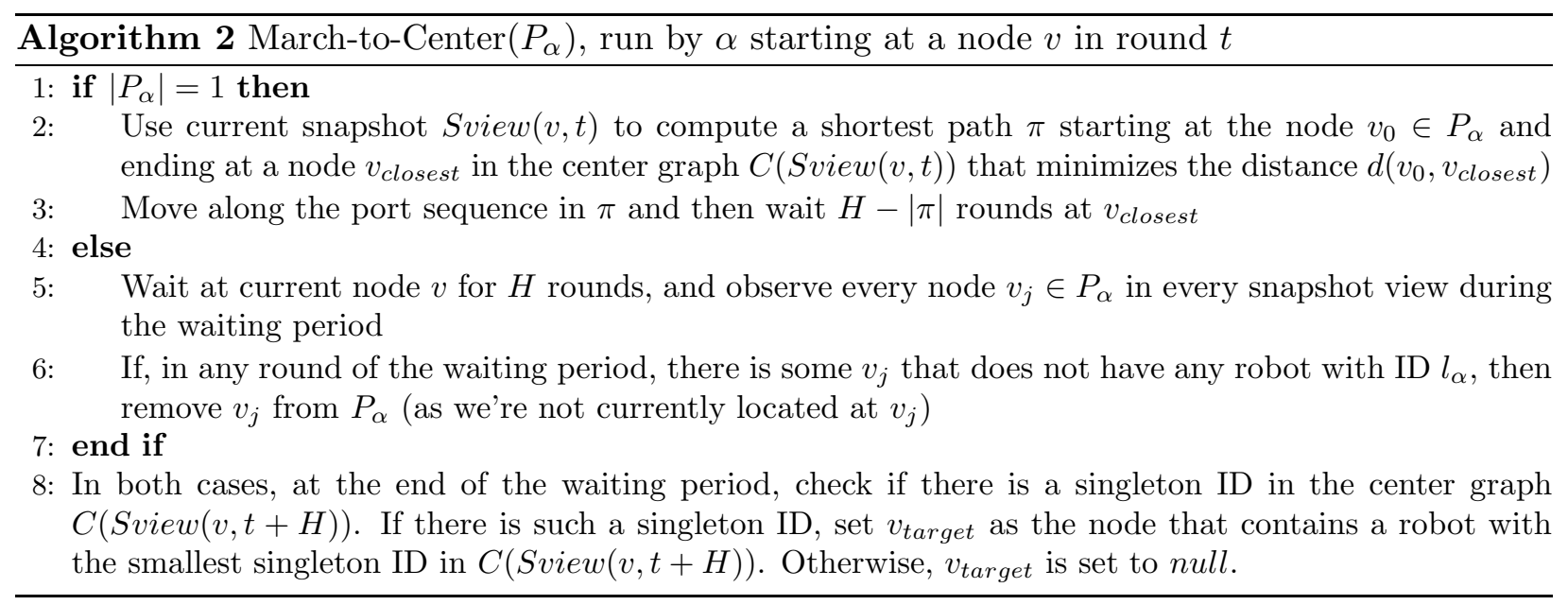

The third part of the algorithm gets each robot to successfully move to the target node $v_{\text {target }}$, which completes the gathering process. This is the purpose of our Merge subroutine. As above, if a robot starts Merge knowing where in its snapshot view it is located (i.e., $\left|P_{\alpha}\right|=1$ ), then it can simply compute a sequence of port numbers that leads to $v_{\text {target }}$ and follow it. The difficult case is when a robot $\alpha$ is not sure where in its snapshot it is located at the start of Merge (i.e., $\left|P_{\alpha}\right|>1$ ). In this case, $\alpha$ just tries one node from its list of possibilities, computes a sequence of port numbers that leads to $v_{\text {target }}$, and tries to follow it. If it notices any inconsistencies along the way or after it arrives, it deletes the guessed starting node from its list $P_{\alpha}$. After each Merge, each robot reverses the steps it took during the Merge in order to go back to where it started so that it can run Merge again. Each execution of Merge finishes in one of two ways: all robots have gathered, or, at least one robot has eliminated one incorrect guess about its starting position. So, after a carefully chosen number of repetitions, we can guarantee that the last performed Merge gathers all robots at the same node. See Algorithm 3 for the pseudocode of Merge.

The pseudocode for the complete algorithm, called the H-View-Algorithm, is provided as Algorithm 4 .

\subsection{Analysis}

We consider three main parts of the algorithm. Our first goal is to show that, immediately after robot $\alpha$ executes Find-Lookout, it has moved to a node $v_{\max }$ such that the snapshot view from 

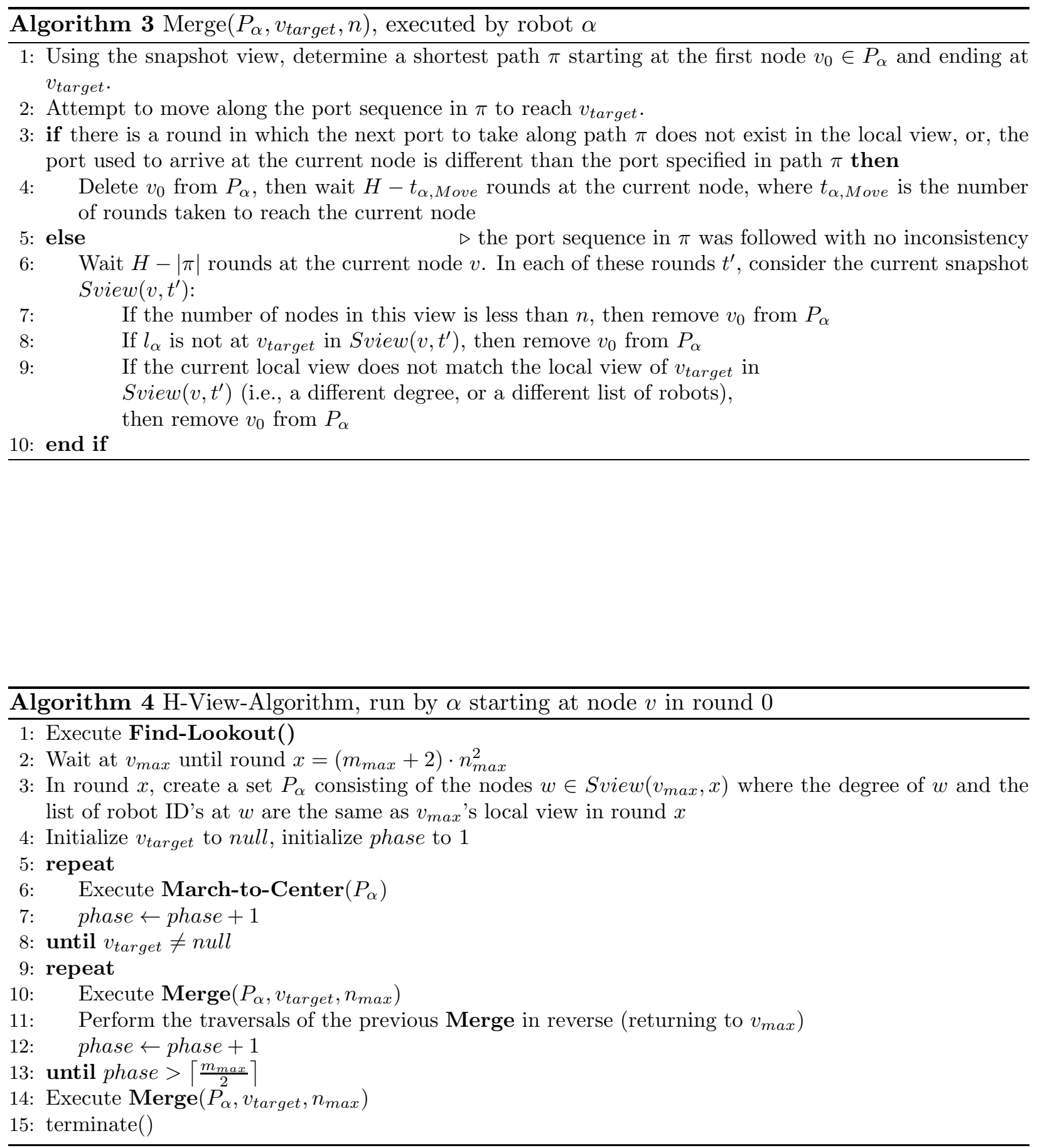
$v_{\max }$ contains $n_{\max }=n$ nodes and $m_{\max }=m$ robots.

Lemma 2.1. By round $(m+2) \cdot n^{2}$, each non-faulty robot $\alpha$ is located at a node $v_{\max }$ such that the snapshot view at $v_{\max }$ contains $n$ nodes and $m$ robots.

Proof. Consider an arbitrary robot $\alpha$ 's execution of the H-View-Algorithm starting at a node $v$. First, $\alpha$ computes the set of nodes $w \in \operatorname{Sview}(v, 0)$ where the degree of $w$ and the list of robot ID's at $w$ is the same as $v$ 's local view in round 0 . In particular, this means that each such node $w$ contains $\alpha$ 's ID $l_{\alpha}$ in its list of robots. Since at most $f+1$ robots can have ID $l_{\alpha}$ in round 0 (i.e., $\alpha$ itself and at most $f$ Byzantine robots), we get that the number of nodes $w$ in Sview $(v, 0)$ that look the same as $\operatorname{Lview}(v, 0)$ is at most $f+1$. Consequently, this means that the number of different depth-first traversals attempted by $\alpha$ is at most $f+1$. Each depth-first traversal takes at most $2|E|$ rounds, which is less than $n^{2}$. Together with the reversal to return back to its starting node, we get that each attempt takes at most $2 n^{2}$ rounds, so all traversals are complete by round $2(f+1) \cdot n^{2}$. Since one of the computed traversal sequences starts at $\alpha$ 's real initial location, it follows that at least one of the traversal attempts visits all nodes in Sview $(v, 0)$. By the definition of the network's center and the fact that $H \geq R$, it follows that $S v i e w(v, 0)$ must contain a node that is in the network's center, and we just showed that $\alpha$ necessarily visited all nodes in Sview $(v, 0)$. Since the snapshot view at any node in the center of the network contains all of the network's nodes (since $H \geq R$ ), it follows that $\alpha$ visits at least one node at which the snapshot view contains all $n$ nodes (and contains all $m$ robots). Robot $\alpha$ will save such a node as $v_{\max }$, it will set $n_{\max }=n$ and $m_{\max }=m$, and it will set $\tau_{\max }$ to be a port sequence from $v$ to $v_{\max }$. The final traversal of the path $\tau_{\max }$ to get from $v$ to $v_{\max }$ takes at most another $n^{2}$ rounds, so, in total, $\alpha$ arrives at $v_{\max }$ by round $(2 f+3) \cdot n^{2}$. Since the number of non-faulty robots is at least $f+1$, we get that $m \geq 2 f+1$, so $f \leq \frac{m-1}{2}$. Thus, $(2 f+3) \cdot n^{2} \leq(m+2) \cdot n^{2}$.

The second part of the algorithm consists of the executions of March-to-Center. Our main goal is to prove that, after at most $f+1$ executions of March-to-Center, every robot sets its $v_{\text {target }}$ variable to the same non-null value. To this end, we first prove that each execution of March-toCenter by the non-faulty robots is started at the same time, and, at the end of each execution, every robot is located at a node such that its snapshot contains all of the network's nodes. This allows us to conclude that any particular feature seen by one robot can be seen by all other robots at the same time.

Lemma 2.2. At the end of each execution of March-to-Center by any non-faulty robot $\alpha$, the robot resides at some node $v$ such that its snapshot view contains all the nodes of $G$.

Proof. We consider the two cases in the description of March-to-Center. We note that, at the end of each execution of March-to-Center by a non-faulty robot $\alpha$, either $\alpha$ is at the node $v_{\max }$ where it started the execution, or, it is at a node $v_{\text {closest }}$ which is defined to be in $C\left(\operatorname{Sview}\left(v_{\max }, t\right)\right)$, i.e., the center graph of $\alpha$ 's snapshot view from node $v_{\max }$. In the first case, Lemma 2.1 tells us that the snapshot view from node $v_{\max }$ contains all the nodes of $G$. In the second case, we observe that $v_{\text {closest }}$ is in the center of $G$ since it is in the center graph of $\alpha$ 's snapshot view from node $v_{\max }$ (which contains all nodes of $G$ ). But by the definition of center, the distance from $v_{\text {closest }}$ to any node in $G$ is at most $R \leq H$, so all nodes of $G$ are in the snapshot view from $v_{\text {closest }}$ as well.

Lemma 2.3. Suppose that every non-faulty robot starts an execution of March-to-Center in the same round $t^{\prime}>0$. In round $t^{\prime}+H$, every non-faulty robot has the same snapshot view. 
Proof. We see from the description of March-to-Center that there can be two cases in each execution: moving along the path $\pi$ for $|\pi|$ rounds followed by a waiting period of length $H-|\pi|$, or, a waiting period of length $H$. In both cases, the execution takes exactly $H$ rounds. Now, by Lemma 2.2, we see that at the end of the execution, i.e., in round $t^{\prime}+H$, each robot's snapshot view is the entire graph.

Lemma 2.4. For any positive integers $i$ and $t^{\prime}$, suppose that every non-faulty robot starts its $i^{\text {th }}$ execution of March-to-Center in round $t^{\prime}$. Then, at the start of round $t^{\prime}+H$, exactly one of the following is true: (i) every non-faulty robot sets vtarget equal to a non-null value, or, (ii) every nonfaulty robot has $v_{\text {target }}$ equal to null, and they all start their $(i+1)^{\text {th }}$ execution of March-to-Center.

Proof. By Lemma 2.3, in round $t^{\prime}+H$, every robot gets the same snapshot view $S$. There are two cases to consider. In the first case, suppose that there is a singleton ID in the center graph of $S$. Then, according to the description of March-to-Center, every non-faulty robot sets its variable $v_{\text {target }}$ to the node that contains a robot with the smallest singleton ID, which implies that every non-faulty robot has $v_{\text {target }}$ equal to a non-null value. In the second case, suppose that there is no singleton ID in the center graph of $S$. Then, according to the description of March-to-Center, $v_{\text {target }}$ at each non-faulty robot remains null. According to the description of the H-View-Algorithm, this means that all non-faulty robots will execute March-to-Center again.

We now proceed to show that each execution of March-to-Center by the non-faulty robots is started at the same time. This is useful because it means that the robots make decisions using the same snapshot view, which minimizes the influence of the Byzantine robots: if a Byzantine robot imitates a non-faulty robot's ID $l$ in a fixed round $t$, then it cannot imitate any other ID's in the same round.

Lemma 2.5. For any positive integer $k$, suppose that all non-faulty robots start their $k^{\text {th }}$ execution of March-to-Center and have $v_{\text {target }}=$ null. For every positive integer $i \leq k$, every non-faulty robot starts executing its $i^{\text {th }}$ execution of March-to-Center in round $(m+2) n^{2}+(i-1) H$.

Proof. We prove the statement by induction on $i$.

Base case: From the description of the H-View-Algorithm, each non-faulty robot executes March-to-Center for the first time starting in round $\left(m_{\max }+2\right) \cdot n_{\max }^{2}=(m+2) \cdot n^{2}$. Thus, the statement is true for $i=1$.

Inductive step: Assume that, for some $j \in\{1, \ldots, k-1\}$, the statement is true for $i=$ $j$. In particular, assume that every robot started its $j^{\text {th }}$ execution of March-to-Center in round $(m+2) n^{2}+(j-1) H$. By the description of March-to-Center, there can be two cases in their $j^{t h}$ execution: moving along the path $\pi$ for $|\pi|$ rounds followed by a waiting period of length $H-|\pi|$, or, a waiting period of length $H$. In both cases, the execution takes exactly $H$ rounds. By Lemma 2.4 and the fact that no robot has set its $v_{\text {target }}$ variable to a non-null value before the $k^{\text {th }}$ execution, we get that in round $(m+2) n^{2}+(j-1) H+H=(m+2) n^{2}+j \cdot H$, every robot starts its $(j+1)^{t h}$ execution of March-to-Center.

Lemma 2.6. Let $k>0$ be the smallest integer such that at least one non-faulty robot sets its $v_{\text {target }}$ to a non-null value during its $k^{\text {th }}$ execution of March-to-Center, and suppose that this execution of March-to-Center starts in round $t^{\prime}$. Then, every non-faulty robot sets $v_{\text {target }}$ to the same value at the start of round $t^{\prime}+H$. 
Proof. By Lemma 2.5. for every positive integer $i \leq k$, every robot starts its $i^{t h}$ execution of Marchto-Center in the same round, so all robots start the $k^{\text {th }}$ execution of March-to-Center in round $t^{\prime}$. Lemma 2.4 implies that, at the start of round $t^{\prime}+H$, either every robot sets a non-null value of $v_{\text {target }}$, or, variable $v_{\text {target }}$ is null for every robot. The second case does not occur since we know that at least one non-faulty robot sets its $v_{\text {target }}$ to a non-null value during its $k^{\text {th }}$ execution of March-to-Center. Therefore, the first case occurs: all robots set their $v_{\text {target }}$ to a non-null value at the start of round $t^{\prime}+H$. Moreover, by Lemma 2.3. every robot has the same snapshot view $S$ in round $t^{\prime}+H$. Hence, by the description of March-to-Center, every robot sets its $v_{\text {target }}$ to the same node: the node that contains a robot with smallest singleton ID in the center graph of $S$.

Corollary 2.7. If there exists a positive integer $k$ such that at least one non-faulty robot sets its $v_{\text {target }}$ to a non-null value during its $k^{\text {th }}$ execution of March-to-Center, then all non-faulty robots set $v_{\text {target }}$ to the same non-null value at the start of round $(m+2) n^{2}+k H$.

We now set out to show that all robots set their $v_{\text {target }}$ variable to a non-null value within $f+1$ executions of March-to-Center. The idea behind the proof is to show that, in each execution of March-to-Center that ends with $v_{\text {target }}=$ null, at least one non-faulty robot makes progress towards determining its correct location within its snapshot view. Once there are enough robots that have determined their correct location (more than the number of Byzantine robots), we are guaranteed to have at least one singleton ID appear in the center of the graph, and all robots will set their $v_{\text {target }}$ as the location of the smallest such ID.

To formalize the argument, we introduce a function $\Phi$ that measures how much progress has been made by all robots towards determining their correct location within their snapshot view. In what follows, for each $t \geq(m+2) \cdot n^{2}$, we denote by $P_{\alpha, t}$ the value of variable $P_{\alpha}$ at robot $\alpha$ in round $t$. From the description of the H-View-Algorithm, recall that $P_{\alpha}$ is set by each robot $\alpha$ for the first time in round $(m+2) \cdot n^{2}$, and the value assigned in this round is the set of nodes in $\alpha$ 's snapshot view that match its local view, i.e., the nodes that have the same degree and the same list of robot ID's as $\alpha$ 's current location. In subsequent rounds, the only changes to $P_{\alpha}$ involve the removal of nodes, so $P_{\alpha, t+1} \subseteq P_{\alpha, t}$ for all $t>(m+2) \cdot n^{2}$. For any fixed round $t \geq(m+2) \cdot n^{2}$, we denote by $\Phi_{t}$ the sum $\sum_{\alpha}\left|P_{\alpha, t}\right|$, which is taken over all non-faulty robots $\alpha$. We now prove some useful bounds on $\Phi_{t}$ and how its value changes in each execution of March-To-Center.

Proposition 2.8. In any round $t \geq(m+2) \cdot n^{2}$, we have $m-f \leq \Phi_{t} \leq m$.

Proof. First, we show that $\Phi_{t} \leq m$. Since each $P_{\alpha, t}$ only contains nodes where the ID $l_{\alpha}$ appears in round $t$, it follows that $\left|P_{\alpha, t}\right|$ is bounded above by the number of robots whose ID in round $t$ is equal $l_{\alpha}$. As each robot has exactly one ID in round $t$ (including the Byzantine robots), it follows that $\Phi_{t}=\sum_{\alpha}\left|P_{\alpha, t}\right| \leq m$. Next, to show that $\Phi_{t} \geq m-f$, we observe that there are $m-f$ non-faulty robots, and each non-faulty robot $\alpha$ has $\left|P_{\alpha, t}\right| \geq 1$ in every round $t \geq(m+2) \cdot n^{2}$. This is because a non-faulty robot $\alpha$ only removes a node $v$ from $P_{\alpha}$ if it performs March-to-Center or Merge under the assumption that it starts the execution from node $v$ in its snapshot view, but notices an inconsistency between this assumption and its observed experience. Since $\alpha$ 's actual $v_{\max }$ node from which it starts March-to-Center or Merge would not result in any inconsistency, this node would never be removed from $P_{\alpha}$, which implies that $\left|P_{\alpha}\right| \geq 1$ after the first round in which $P_{\alpha}$ is given a value. 
Lemma 2.9. Consider any execution of March-to-Center by the non-faulty nodes, and suppose that the execution starts in round $t^{\prime}$. Then, exactly one of the following occurs: (i) all non-faulty robots set their vtarget variable to a non-null value at the start of round $t^{\prime}+H$, or, (ii) $\Phi_{t^{\prime}+H} \leq \Phi_{t^{\prime}}-1$.

Proof. By Lemma 2.4, exactly one of the following occurs at the start of round $t^{\prime}+H$ :

- All non-faulty robots set their $v_{\text {target }}$ variable to some non-null value, or,

- Variable $v_{\text {target }}$ is null for every robot. By Lemma 2.3, we know that in round $t+H^{\prime}$, all non-faulty robots have the same snapshot view $S$, and, by Lemma 2.2, $S$ contains all the nodes of $G$. As there are at least $f+1$ non-faulty robots and exactly $f$ Byzantine robots, there must be at least one non-faulty robot $\beta$ whose ID will be a singleton ID in $S$. But since $v_{\text {target }}$ is null for every non-faulty robot, this implies that there is no singleton ID in $C(S)$ in round $t^{\prime}+H$, and so $\beta$ is located outside of $C(S)$. According to the description of March-to-Center, it must be the case that $\left|P_{\beta}\right|>1$ in round $t^{\prime}$, because otherwise $\beta$ would have moved to a node in the center of its snapshot view in this execution of March-to-Center. Consequently, according to March-to-Center, the robot $\beta$ removes all other nodes from $P_{\beta}$ except the one node that contains its ID $l_{\beta}$ (as $l_{\beta}$ is a singleton ID). Thus, the value of $\left|P_{\beta}\right|$ decreases during some round in the range $t^{\prime}, \ldots, t^{\prime}+H$, so it follows that $\Phi_{t^{\prime}+H} \leq \Phi_{t^{\prime}}-1$.

Theorem 2.10. There exists a positive integer $k \leq f+1$ such that every non-faulty robot sets its variable $v_{\text {target }}$ to the same non-null value at the start of round $(m+2) n^{2}+k H$.

Proof. First, suppose that there is at least one non-faulty robot that sets its $v_{\text {target }}$ to a nonnull value during one of its first $f$ executions of March-to-Center. In this case, the desired result follows directly from Corollary 2.7. So, in what follows, we assume that all non-faulty robots have $v_{\text {target }}=$ null during the first $f$ executions of March-to-Center. Therefore, all non-faulty robots start their $(f+1)^{t h}$ execution of March-to-Center with $v_{\text {target }}=$ null, and by Lemma 2.5, they start this execution in round $(m+2) n^{2}+f H$. By Lemmas 2.2 and 2.3, each non-faulty robot starts this execution with the same snapshot view, which we'll denote by $S$, that contains all the nodes of $G$.

By Lemma 2.9, after each of the first $f$ executions of March-to-Center, the value of $\Phi$ decreases by at least 1. It follows that $\Phi_{(m+2) n^{2}+f H} \leq \Phi_{(m+2) n^{2}}-f$. However, by Proposition 2.8, we know that $\Phi_{(m+2) n^{2}} \leq m$ and $\Phi_{(m+2) n^{2}+f H} \geq m-f$, so altogether we conclude that $\Phi_{(m+2) n^{2}+f H}=m-f$. But $m-f$ is the number of non-faulty robots, so the sum $\Phi_{(m+2) n^{2}+f H}=\sum_{\alpha}\left|P_{\alpha,(m+2) n^{2}+f H}\right|$ has $m-f$ non-zero terms. This implies that each $\left|P_{\alpha,(m+2) n^{2}+f H}\right|$ is equal to exactly 1 . Therefore, by the description of March-to-Center, all non-faulty robots move to a node in the center graph of their snapshot view $S$. This means that there are at least $f+1$ non-faulty robots in the center of $S$ in round $(m+2) n^{2}+(f+1) H$, and at least one of their ID's is a singleton ID since there are at most $f$ Byzantine nodes. Thus, by the description of March-to-Center, every non-faulty robot sets its $v_{\text {target }}$ to the same node: the node that contains a robot with smallest singleton ID in the center graph of $S$, which proves the desired statement with $k=f+1$.

Now we come to the third part of the algorithm which consists of the executions of Merge. By the description of the H-View-Algorithm, non-faulty robots start executing their Merge operation immediately after setting a non-null value of $v_{\text {target }}$. Moreover, by Theorem 2.10 , we see that every 
robot sets its $v_{\text {target }}$ variable to the same non-null value in the same round, and so every non-faulty robot starts executing its first execution of Merge at the same time as well. More specifically, we denote by $k$ the number of executions of March-to-Center performed by the non-faulty robots, and conclude that all non-faulty robots start their first execution of Merge in round $(m+2) n^{2}+k H$. By the description of Merge, each execution of Merge consists of exactly $H$ rounds, and according to the $\mathrm{H}-\mathrm{View}-\mathrm{Algorithm}$, an additional $H$ rounds are then used to perform the steps of Merge in reverse. These observations imply the following fact.

Lemma 2.11. For any positive integer $i$, if an $i^{\text {th }}$ execution of Merge is performed, then all nonfaulty robots start this execution in round $(m+2) n^{2}+(k+2(i-1)) H$.

Our final goal is to show that all non-faulty robots gather at $v_{\text {target }}$ after at most $(f+2)-k$ executions of Merge, where $k$ is the number of March-to-Center operations executed by the nonfaulty robots. Before proving this in Theorem 2.15, we establish the following technical results.

Lemma 2.12. For any $t \geq 0$, suppose that $v$ is a node such that at least $m-f$ robots are located at $v$ at the start of round $t$. Then, the local view at $v$ in round $t$ is unique. More precisely, for any node $v^{\prime} \neq v$, we have Lview $\left(v^{\prime}, t\right) \neq \operatorname{Lview}(v, t)$.

Proof. For any $v, v^{\prime}$ such that $v \neq v^{\prime}$, if there are at least $m-f$ robots at $v$ in round $t$, there can be at most $f$ robots at $v^{\prime}$ in round $t$. Since there are at least $f+1$ non-faulty robots, it follows that $m \geq 2 f+1$, so $m-f>f$. In particular, this means that the number of ID's in Lview $(v, t)$ is strictly greater than the number of ID's in $\operatorname{Lview}\left(v^{\prime}, t\right)$, so $\operatorname{Lview}(v, t) \neq \operatorname{Lview}\left(v^{\prime}, t\right)$.

Lemma 2.13. Consider any execution of Merge by the non-faulty nodes, and suppose that the execution starts in round $t^{\prime}$. Then at least one of the following holds: (i) all non-faulty robots are gathered at virget in round $t^{\prime}+H$, or, (ii) $\Phi_{t^{\prime}+H} \leq \Phi_{t^{\prime}}-1$.

Proof. Assume that (i) does not hold in round $t^{\prime}+H$, i.e., at least one non-faulty robot is not located at $v_{\text {target }}$ in round $t^{\prime}+H$. There are two possibilities:

- There are at least $m-f$ robots at $v_{\text {target }}$ in round $t^{\prime}+H$. By Lemma 2.12, each robot $\beta$ that is at a node $v^{\prime} \neq v_{\text {target }}$ in round $t^{\prime}+H$ has a local view Lview $\left(v^{\prime}, t^{\prime}+H\right)$ that is different than Lview $\left(v_{\text {target }}, t^{\prime}+H\right)$. Hence, according to the description of Merge, each such robot $\beta$ removes a node from its $P_{\beta}$, i.e., the value of $\left|P_{\beta}\right|$ decreases in some round in the range $t^{\prime}, \ldots, t^{\prime}+H$. It follows that $\Phi_{t^{\prime}+H} \leq \Phi_{t^{\prime}}-1$.

- There are fewer than $m-f$ robots at $v_{\text {target }}$ in round $t^{\prime}+H$. As the number of nonfaulty robots is $m-f$, it follows that there is at least one non-faulty robot $\alpha$ whose ID $l_{\alpha}$ is not seen at $v_{\text {target }}$ in $\alpha$ 's snapshot view in round $t^{\prime}+H$. Hence, according to the description of Merge, $\alpha$ removes a node from its $P_{\alpha}$, i.e., the value of $\left|P_{\alpha}\right|$ decreases in some round in the range $t^{\prime}, \ldots, t^{\prime}+H$. It follows that $\Phi_{t^{\prime}+H} \leq \Phi_{t^{\prime}}-1$.

Lemma 2.14. During the execution of the H-View-Algorithm, if $k \geq 1$ executions of March-toCenter are performed followed by $f+2-k$ executions of Merge, then all non-faulty robots are gathered at virget. 
Proof. By the description of the H-View-Algorithm and Corollary 1, if $k$ executions of March-toCenter are performed, then $v_{\text {target }}$ was set for the first time by all non-faulty robots at the end of the $k^{\text {th }}$ execution of March-to-Center. By Lemma 2.9, after each of the first $k-1$ executions of March-toCenter, the value of $\Phi$ decreases by at least 1 . It follows that $\Phi_{(m+2) n^{2}+(k-1) H} \leq \Phi_{(m+2) n^{2}}-(k-1)$. By Proposition 2.8, we know that $\Phi_{(m+2) n^{2}} \leq m$, so it follows that $\Phi_{(m+2) n^{2}+(k-1) H} \leq m-(k-1)$. Since the value of $\Phi$ never increases (the algorithm only ever removes nodes from the $P_{\alpha}$ sets) it follows that $\Phi_{(m+2) n^{2}+k H} \leq m-(k-1)$ as well, where round $(m+2) n^{2}+k H$ is when the first Merge execution begins. Now, we consider the first $f+1-k$ executions of Merge by the non-faulty robots, and we consider two cases:

- Suppose that, for some $i \in\{1, \ldots, f+1-k\}$, all non-faulty robots are gathered at $v_{\text {target }}$ at the end of the $i^{t h}$ execution of Merge. Since the number of non-faulty robots is $m-f$, it follows that there would be at least $m-f$ robots at $v_{\text {target }}$. By Lemma 2.12, the local view at $v_{\text {target }}$ would be unique in $G$, and the local view of each non-faulty robot would exactly match it. Hence, according to the description of Merge, no non-faulty robot would modify its $P_{\alpha}$ set, and so the next execution of Merge (if any) would start from the same node $v_{0}$. It follows that in all subsequent executions of Merge (in particular, the $(f+2-k)^{t h}$ execution) all non-faulty robots will be gathered at $v_{\text {target }}$.

- Suppose that, for every $i \in\{1, \ldots, f+1-k\}$, at least one non-faulty robot is not located at $v_{\text {target }}$ at the end of the $i^{\text {th }}$ execution of Merge. Then, according to Lemma 2.13, the value of $\Phi$ decreases by at least 1 in each such execution. As the value of $\Phi$ was bounded above by $m-(k-1)$ at the start of the first Merge execution, and it decreases by at least $f+1-k$ during the first $f+1-k$ executions of Merge, it follows that, after the $(f+1-k)^{t h}$ execution of Merge, the value of $\Phi$ is at most $m-f$. However, by Proposition 2.8, we know that $\Phi$ is at least $m-f$, so altogether we conclude that the value of $\Phi$ after the $(f+1-k)^{t h}$ execution of Merge is exactly $m-f$. But $m-f$ is the number of non-faulty robots, so the summation represented by $\Phi$ has $m-f$ non-zero terms. This implies that each $\left|P_{\alpha}\right|$ is equal to exactly 1 for each non-faulty robot $\alpha$. Then, in the final execution of Merge, i.e., in execution $f+2-k$, each non-faulty robot will compute a path to vtarget using its snapshot view, but using its actual location as starting node $v_{0}$. This means that all non-faulty nodes will be located at $v_{\text {target }}$ at the end of execution $f+2-k$ of Merge.

Finally, we verify that the $\mathrm{H}$-View-Algorithm ensures that Merge is executed at least $f+2-k$ times after $k$ executions of March-to-Center. The Merge operation is executed until the value of phase is greater than $\lceil m / 2\rceil$, and from the assumption that the number of non-faulty robots is at least $f+1$, we know that $m \geq 2 f+1$. In particular, this means that the combined number of March-to-Center and Merge executions is at least $f+1$, and then one more Merge is executed after exiting the 'repeat' loop. This concludes the proof of correctness of the H-View-Algorithm.

Theorem 2.15. In any $n$-node graph with radius $R$, if the $H$-View-Algorithm is performed by any team of $m$ robots consisting of $f$ Byzantine robots and at least $f+1$ non-faulty robots with visibility $H \geq R$, then Gathering is solved within $(m+2) \cdot n^{2}+H \cdot m \in O\left(m n^{2}\right)$ rounds.

Proof. By Lemma 2.1, every non-faulty robot spends exactly $(m+2) n^{2}$ rounds for the FindLookout operation. Then, by Theorem 2.10, there exists a positive integer $k \leq f+1$ such that 
every non-faulty robot sets its variable $v_{\text {target }}$ at the start of the round $(m+2) n^{2}+k H$. More precisely, robots spend exactly $k H$ rounds performing the March-to-Center executions. After that, every robot spends exactly $(\lceil m / 2\rceil-k) 2 H+H$ rounds for its Merge executions, after which all non-faulty are located at $v_{\text {target }}$ (by Lemma 2.14, In total, the number of rounds is $(m+2) \cdot n^{2}+k H+(\lceil m / 2\rceil-k) 2 H+H$. For the minimum value of $k=1$, we get that the robots use at most $(m+2) \cdot n^{2}+H \cdot m$ rounds to accomplish the gathering. As $H \leq n$ (at most full visibility), the number of rounds is in $O\left(m n^{2}\right)$, i.e., polynomial in the network size and team size.

\section{Impossibility Results}

First, we recall Theorem 4.7 from [10], which states that there is no deterministic algorithm that solves Gathering in the presence of $f$ Byzantine robots if the number of non-faulty agents is at most $f$ (and these non-faulty agents know the size of the graph). This impossibility result was proven in a model where robots have no visibility beyond their local view (i.e., visibility $H=0$ ). However, the same proof works under the assumption that each non-faulty robot has full visibility of the entire graph in every round, which proves that our algorithm is optimal with respect to the number of non-faulty robots in the team.

Theorem 3.1. There is no deterministic algorithm that solves Gathering if the number of Byzantine robots in the team is $f$ and the number of non-faulty robots is at most $f$, even if the non-faulty agents have visibility $H$ equal to the diameter of the graph.

Next, we prove that to solve Gathering in arbitrary graphs, the visibility $H$ of each non-faulty robot must somehow depend on the radius of the graph. In particular, it is not sufficient to fix some constant visibility range. We remark that this does not contradict the existence of previously-known algorithms that work when $H=0$, as those algorithms make additional assumptions that are not present in our model (e.g., knowledge of the graph size, knowledge of the number of Byzantine robots, or whiteboards at the nodes).

Theorem 3.2. There is no deterministic algorithm that can solve Gathering when executed in any graph by any team of $m$ robots consisting of $f \geq 0$ Byzantine robots and at least $f+1$ non-faulty robots if the visibility range $H$ of each non-faulty robot is a fixed constant $c$.

Proof. Let $c$ be any fixed positive integer. To obtain a contradiction, assume the existence of a deterministic algorithm $A$ that can solve Gathering when executed in any graph by any team of $m$ robots consisting of $f \geq 0$ Byzantine robots and at least $f+1$ non-faulty robots if the visibility range $H$ of each non-faulty robot is equal to $c$.

First, we construct an instance consisting of a cycle graph $C_{1}=\left(V_{1}, E_{1}\right)$ with an even number of nodes $\left|V_{1}\right|=2 c+2$. The radius $R_{1}$ of $C_{1}$ is $c+1$. At each node $v \in V_{1}$, the two incident edges are labeled with port numbers 0 and 1 such that 0 leads clockwise and 1 leads anticlockwise. The initial positions of the robots in $C_{1}$ are as follows: a non-faulty robot $\alpha$ with ID $l_{\alpha}$ is placed at some node $v_{0}$, and a non-faulty robot $\beta$ with ID $l_{\beta}$ at a node $w$ such that the distance $d\left(v_{0}, w\right)=R_{1}=c+1$. There are no Byzantine robots in $C_{1}$. Consider the execution $E X_{1}$ of algorithm $A$ on instance $C_{1}$. As $A$ is assumed to be a correct algorithm, there exists some round $r_{1}$ in which robots $\alpha$ and $\beta$ have terminated and gathered at some node $v_{\text {target }} \in V_{1}$.

Next, we construct a second instance consisting of a cycle graph $C_{2}=\left(V_{2}, E_{2}\right)$ with an even number of nodes $\left|V_{2}\right|=4 r_{1}+2(c+1)$. The radius of $R_{2}$ of $C_{2}$ is $2 r_{1}+c+1$. At each node $v \in V_{2}$, 
the two incident edges are labeled with port numbers 0 and 1 such that 0 leads clockwise and 1 leads anticlockwise. The initial positions of the robots in $C_{2}$ are as follows: the non-faulty robot $\alpha$ with ID $l_{\alpha}$ is placed at node $v_{0}$ (as in the first instance $C_{1}$ above), a Byzantine robot with ID $l_{\beta}$ is placed at a node $v_{C W}$ that is distance exactly $c+1$ away from $v_{0}$ in the clockwise direction, and another Byzantine robot with ID $l_{\beta}$ is placed at a node $v_{A C W}$ that is distance exactly $c+1$ away from $v_{0}$ in the anticlockwise direction. Further, we place 2 non-faulty robots at a node $w$ such that $d\left(v_{0}, w\right)=R_{2}=2 r_{1}+c+1$. These 2 non-faulty robots have distinct ID's that are not equal to $l_{\alpha}$ or $l_{\beta}$. The number of Byzantine robots is $f=2$, and there are $3=f+1$ non-faulty robots (one at $v_{0}$ and two at $\left.w\right)$. We denote by $E X_{2}$ the execution of algorithm $A$ on instance $C_{2}$.

We now demonstrate that the Byzantine robots in $C_{2}$ can behave in such a way that, for each round $t$, the robot $\alpha$ with ID $l_{\alpha}$ cannot distinguish between executions $E X_{1}$ and $E X_{2}$, i.e., robot $\alpha$ 's local view and snapshot view in every round are the same across both executions. This leads to a contradiction: since $\alpha$ terminates its algorithm in round $r_{1}$ in execution $E X_{1}$, it will also terminate its algorithm in round $r_{1}$ in execution $E X_{2}$, and since the initial distance between $\alpha$ and the other non-faulty robots is strictly greater than $2 r_{1}$, it follows that $\alpha$ terminates before the non-faulty robots can gather.

First, note that $\alpha$ 's visibility range is $c$ in both executions, which means that its snapshot view consists of $2 c+1$ nodes in every round of both executions. By the initial placement of the robots in both executions, we note that in round $t=0$ of both executions, there are no robots within distance $c$ of $\alpha$ 's initial position $v_{0}$. So, $\alpha$ 's local view in round 0 of both executions consists of a node of degree 2 containing the ID $l_{\alpha}$, and, $\alpha$ 's snapshot view in round 0 of both executions consists of a path of length $2 c+1$ nodes with only ID $l_{\alpha}$ located at the middle node. Further, we note that the two other non-faulty robots in $C_{2}$ are never visible to $\alpha$ in execution $E X_{2}$ : their initial distance to $\alpha$ is $2 r_{1}+c+1$, so in round $r_{1}$, each of their distances to $\alpha$ is at least $c+1$.

To define the behaviour of the Byzantine robots in $C_{2}$ during rounds $t=1, \ldots, r_{1}$ of execution $E X_{2}$, we observe the execution $E X_{1}$. In particular:

- For each round $t>0$ of $E X_{1}$ in which $\alpha$ does not see $\beta$ in its snapshot view: the Byzantine robots follow the same port in round $t-1$ of $E X_{2}$ as $\alpha$ did in round $t-1$ in $E X_{1}$. Doing so ensures that both Byzantine robots remain at distance $c+1$ from $\alpha$ at the start of round $t$ in $E X_{2}$, i.e., are not in $\alpha$ 's snapshot view.

- For each round $t>0$ of $E X_{1}$ in which $\alpha$ sees $\beta$ in its snapshot view but did not see $\beta$ in its snapshot view in round $t-1$ : the Byzantine robot on the appropriate side of $\alpha$ (clockwise or counterclockwise) moves so that it appears at the same node in $\alpha$ 's snapshot view in round $t$ of $E X_{2}$ as $\beta$ does in round $t$ of $E X_{1}$. The other Byzantine robot follows the same port as $\alpha$ does in round $t-1$ (so that its distance from $\alpha$ at the start of round $t$ is still $c+1$, i.e., it does not appear in $\alpha$ 's snapshot view).

- For each round $t>0$ of $E X_{1}$ in which $\alpha$ sees $\beta$ in its snapshot view and also saw $\beta$ in its snapshot view in round $t-1$ : the Byzantine robot that was in $\alpha$ 's snapshot view in round $t-1$ of $E X_{2}$ follows the same port in round $t-1$ of $E X_{2}$ as $\beta$ did in round $t-1$ of $E X_{1}$. The other Byzantine robot follows the same port as $\alpha$ does in round $t-1$ (so that its distance from $\alpha$ at the start of round $t$ is still $c+1$, i.e., it does not appear in $\alpha$ 's snapshot view).

It is clear from this behaviour that $\alpha$ sees the same thing up to round $r_{1}$ in both executions $E X_{1}$ and $E X_{2}$ : when $\alpha$ sees no other robots in round $t$ of $E X_{1}$, then both Byzantine robots move 
so that they are both at distance $c+1$ from $\alpha$ in round $t$ of $E X_{2}$; moreover, when $\alpha$ sees $\beta$ in round $t$ of $E X_{1}$, then one Byzantine robot (which has ID $l_{\beta}$ ) moves so that its position relative to $\alpha$ in round $t$ of $E X_{2}$ is the same as $\beta$ 's relative position to $\alpha$ in round $t$ of $E X_{1}$, while the other Byzantine robot moves so that it is at distance $c+1$ from $\alpha$ in round $t$ of $E X_{2}$.

We were not able to extend the lower bound argument in Theorem 3.2 to a non-constant visibility range $H$. The reason is that, when we change the underlying graph, the visibility radius of a robot is different in the new graph, so we cannot use indistinguishability to conclude that a robot will behave in the same way in both graphs. Establishing a lower bound on $H$ with respect to $R$ is left as an open problem.

\section{References}

[1] Eduardo Mesa Barrameda, Nicola Santoro, Wei Shi, and Najmeh Taleb. Sensor deployment by a robot in an unknown orthogonal region: Achieving full coverage. In 20th IEEE International Conference on Parallel and Distributed Systems, ICPADS 2014, pages 951-960, 2014.

[2] Lali Barrière, Paola Flocchini, Eduardo Mesa Barrameda, and Nicola Santoro. Uniform scattering of autonomous mobile robots in a grid. Int. J. Found. Comput. Sci., 22(3):679-697, 2011.

[3] Subhash Bhagat, Krishnendu Mukhopadhyaya, and Srabani Mukhopadhyaya. Computation under restricted visibility. In Distributed Computing by Mobile Entities, Current Research in Moving and Computing, pages 134-183. Springer, 2019.

[4] Sébastien Bouchard, Yoann Dieudonné, and Bertrand Ducourthial. Byzantine gathering in networks. Distributed Computing, 29(6):435-457, 2016.

[5] Sébastien Bouchard, Yoann Dieudonné, and Anissa Lamani. Byzantine gathering in polynomial time. In 45th International Colloquium on Automata, Languages, and Programming, ICALP 2018, pages 147:1-147:15, 2018.

[6] Jérémie Chalopin, Yoann Dieudonné, Arnaud Labourel, and Andrzej Pelc. Rendezvous in networks in spite of delay faults. Distributed Computing, 29(3):187-205, 2016.

[7] Jérémie Chalopin, Emmanuel Godard, and Antoine Naudin. Anonymous graph exploration with binoculars. In Distributed Computing - 29th International Symposium, DISC 2015, pages 107-122, 2015.

[8] Serafino Cicerone, Gabriele Di Stefano, and Alfredo Navarra. Asynchronous robots on graphs: Gathering. In Distributed Computing by Mobile Entities, Current Research in Moving and Computing, pages 184-217. Springer, 2019.

[9] Xavier Défago, Maria Potop-Butucaru, and Sébastien Tixeuil. Fault-tolerant mobile robots. In Distributed Computing by Mobile Entities, Current Research in Moving and Computing, pages 234-251. Springer, 2019. 
[10] Yoann Dieudonné, Andrzej Pelc, and David Peleg. Gathering despite mischief. ACM Transactions on Algorithms (TALG), 11(1):1, 2014.

[11] Matthias Fischer, Daniel Jung, and Friedhelm Meyer auf der Heide. Gathering anonymous, oblivious robots on a grid. In 13th International Symposium on Algorithms and Experiments for Wireless Sensor Networks, ALGOSENSORS 2017, pages 168-181, 2017.

[12] Paola Flocchini. Gathering. In Distributed Computing by Mobile Entities, Current Research in Moving and Computing, pages 63-82. Springer, 2019.

[13] Paola Flocchini, Giuseppe Prencipe, and Nicola Santoro, editors. Distributed Computing by Mobile Entities, Current Research in Moving and Computing. Springer, 2019.

[14] Jion Hirose, Junya Nakamura, Fukuhito Ooshita, and Michiko Inoue. Gathering with a strong team in weakly byzantine environments. CoRR, abs/2007.08217, 2020.

[15] Tien-Ruey Hsiang, Esther M. Arkin, Michael A. Bender, Sándor P. Fekete, and Joseph S. B. Mitchell. Algorithms for rapidly dispersing robot swarms in unknown environments. In Fifth International Workshop on the Algorithmic Foundations of Robotics, WAFR 2002, pages 7794, 2002.

[16] Fukuhito Ooshita, Ajoy K. Datta, and Toshimitsu Masuzawa. Self-stabilizing rendezvous of synchronous mobile agents in graphs. In Stabilization, Safety, and Security of Distributed Systems - 19th International Symposium, SSS 2017, pages 18-32, 2017.

[17] Andrzej Pelc. Deterministic gathering with crash faults. Networks, 72(2):182-199, 2018.

[18] Andrzej Pelc. Deterministic rendezvous algorithms. In Distributed Computing by Mobile Entities, Current Research in Moving and Computing, pages 423-454. Springer, 2019.

[19] Masashi Tsuchida, Fukuhito Ooshita, and Michiko Inoue. Byzantine-tolerant gathering of mobile agents in arbitrary networks with authenticated whiteboards. IEICE Transactions, 101-D(3):602-610, 2018. 